\title{
Influence of perception of colorectal cancer risk and patient bowel preparation behaviors: a study in minority populations
}

This article was published in the following Dove Press journal:

Clinical and Experimental Gastroenterology

28 January 2015

Number of times this article has been viewed

\author{
Vinaya Gaduputi \\ Chaitanya Chandrala \\ Hassan Tariq \\ Sailaja Sakam \\ Anil Dev \\ Sridhar Chilimuri
}

Bronx Lebanon Hospital Center, Department of Medicine, Bronx, New York, NY, USA
Correspondence: Hassan Tariq Bronx Lebanon Hospital Center, 1650 Selwyn Ave, Suite 10C, Bronx, New York 10457, NY, USA

$\mathrm{Tel}+\mathrm{I} 7189601234$

Fax +l 7189602055

Email htariq@bronxleb.org
Background: Large disparities exist in the utilization rates of screening modalities for colorectal cancer (CRC) in different socioeconomic areas. In this study, we evaluated whether the quality of bowel preparation differed significantly among populations with a high risk of CRC compared with that among the general population after matching for potential confounding factors.

Methods: Hispanic and African American patients who underwent routine screening or surveillance colonoscopies in an outpatient setting between 2003 and 2013 were included in this retrospective study. Patients who underwent colonoscopies for emergent indications and repeat routine screening colonoscopies because of prior history of inadequate bowel preparation were excluded from this study. The patients were divided into three groups: patients having an average risk of being diagnosed with CRC (group 1); patients having a high risk of being diagnosed with CRC because of a personal history of adenomatous polyps (group 2); and patients having a high risk of being diagnosed with $\mathrm{CRC}$ because of a family history of $\mathrm{CRC}$ in first-degree relatives (group 3). All the patients were given preprocedural counseling and written instructions for bowel preparation. Data on demographic information, method of bowel preparation, quality of bowel preparation, comorbidities, and prescription medications were collected.

Results: In all, 834 patients had a "high-risk for CRC" surveillance colonoscopy in view of their personal history of adenomatous polyps and were included in group 2. In total, 250 patients had a "high-risk for CRC" screening colonoscopy in view of their family history of $\mathrm{CRC}$ in first-degree relatives and were included in group 3. Further, 1,000 patients were selected to serve as controls (after matching for age, sex and ethnicity) and were included in group 1. Bowel preparation was graded as good, fair, or poor by the endoscopist performing the study. We observed a significantly higher number of good bowel preparations in group 2 and group 3 ( $P=0.0001$ ) when compared with group 1 (controls) after adjusting for comorbidities and usage of prescription medication that could potentially cause colonic dysmotility. These differences were significant in both Hispanic and African American patients.

Conclusion: Our study showed that perception of CRC risk significantly influenced the bowel preparation behaviors of patients belonging to minority populations, with a significantly greater number of patients with a high risk of CRC having adequate bowel preparations.

Keywords: colorectal cancer, bowel preparation, quality, screening colonoscopy, high-risk populations, minority populations

\section{Introduction}

Colorectal cancer (CRC) is the second most common cause of cancer-related death in the USA. ${ }^{1}$ However, CRC-related mortality has declined by almost $3 \%$ over the past decade. ${ }^{1}$ This decrease has been attributed to improved screening protocols and wider coverage of the population. ${ }^{2}$ Simulation models have shown that the decrease 
in CRC rates is mainly attributable to screening rather than to a decrease in risk factors. ${ }^{3}$ The benefits of colonoscopy in preventing right-sided colon cancers and in decreasing mortality associated with these cancers are unclear., ${ }^{4,5}$ Although a randomized controlled trial did not show any effect of colonoscopy in preventing mortality associated with CRC, long-term observational data have shown that colonoscopy decreases the mortality risk associated with CRC. ${ }^{6}$ Colonoscopy has the highest sensitivity and specificity among all modalities available for CRC screening. It has been postulated that until more reliable data are available from a randomized controlled trial, it is reasonable to expect that use of colonoscopy as a screening modality can reduce CRC-associated mortality rate by $70 \%-80 \%{ }^{7}$

An individual is defined as having a high risk of $\mathrm{CRC}$ if he/she has a personal history of adenomatous polyps, a family history of CRC in a first-degree relative (parent or sibling) before the age of 60 years, or hereditary colon cancer entities such as familial adenomatous polyposis or hereditary nonpolyposis CRC, or a protracted history (more than 8-10 years) of inflammatory bowel disease (ulcerative colitis or Crohn's disease). Adenomatous polyps are detected in up to one third of patients who undergo screening colonoscopy. ${ }^{8}$ These polyps are dysplastic and are hence considered premalignant. Removal of these colonic adenomas is important to minimize the risk of and mortality associated with CRC. A family history of CRC in a first-degree or seconddegree relative is common, and is observed in up to $5 \%$ of the general population aged 20-79 years. ${ }^{9}$ Approximately one quarter of all patients with CRC have a family history of CRC. ${ }^{10}$ It was found in some studies that Hispanic patients had a higher likelihood of undergoing sigmoidoscopy/ colonoscopy while participating in CRC screening when compared with other modalities. ${ }^{11}$ However, to the best of our knowledge, no studies have been performed regarding the quality of bowel preparation among patients with a high risk of $\mathrm{CRC}$ who belong to minority populations.

The most important factor affecting the outcome of colonoscopy is the quality of bowel preparation. Several studies have shown that advanced age, ${ }^{12}$ male sex ${ }^{13,14}$ comorbidities such as diabetes (because of its negative influence on colonic motility due to autonomic neuropathy that leads to impaired postprandial gastrocolic response), ${ }^{15,16}$ and low educational level ${ }^{17,18}$ are independent risk factors for poor bowel preparation. In addition, multiple commonly prescribed medications, such as beta-blockers, calcium channel blockers, opiates, and tricyclic antidepressants, influence bowel motility and thus the quality of bowel preparation.
This retrospective study evaluated whether the quality of bowel preparation differed significantly among populations with a high risk of CRC compared with that among the general population after matching for potential confounding factors. We specifically included patients from minority populations who presented to an inner city hospital because there is paucity of data on factors affecting the quality of bowel preparation in these populations.

\section{Materials and methods \\ Patients}

This retrospective study was performed according to the Declaration of Helsinki. The institutional review board of the study hospital approved the study protocol. Data were collected from electronic medical records of patients and were tabulated in an Excel ${ }^{\circledR}$ spreadsheet (Microsoft Corporation, Redmond, WA, USA). Hispanic and African American patients who underwent routine screening or surveillance colonoscopies in the outpatient setting from 2003 to 2013 were included in this study. All patients received polyethylene glycol-based formulations for bowel preparation, and received routine verbal instructions on bowel preparation and on the procedure itself, with an emphasis on the importance of adherence to the protocol for bowel preparation. In addition, all the patients were provided with instructions printed in their preferred language to remind them about the protocol for bowel preparation. The patients were allowed to consume only a clear liquid diet (fruit juices, lemonades, and tea without milk) on the day before the procedure. In addition, the patients were recommended to maintain a "sip to scope" time (time between the last sip of bowel preparation liquid and colonoscopy) of at least 5 hours. Patients who underwent colonoscopy for emergent indications or while being admitted to the hospital were excluded from the study. The patients included in the study were divided into three groups: patients from the general population who had an average risk of being diagnosed with CRC during screening colonoscopy (group 1); patients who had a high risk of being diagnosed with CRC because of a personal history of adenomatous polyps (group 2); and patients who had a high risk of being diagnosed with CRC because of a family history of CRC in first-degree relatives (group 3).

We collected baseline demographic data, including age, sex and ethnicity, for all the groups. In addition, we collected data on the method of bowel preparation (full-dose or split-dose protocol) and quality of bowel preparation (good, fair, or poor, as graded by endoscopists). We also obtained data on the presence of comorbidities, such as diabetes and 
Table I Baseline characteristics of patients classified based on risk of colorectal cancer before colonoscopy

\begin{tabular}{|c|c|c|c|c|c|}
\hline Characteristic & $\begin{array}{l}\text { Group I } \\
\text { total n, (\%) }\end{array}$ & $\begin{array}{l}\text { Group } 2 \\
\text { total n, (\%) }\end{array}$ & $\begin{array}{l}P \text {-value } \\
\text { Groups I, } 2\end{array}$ & $\begin{array}{l}\text { Group } 3 \\
\text { total n, (\%) }\end{array}$ & $\begin{array}{l}P \text {-value } \\
\text { Groups I, } 3\end{array}$ \\
\hline Age, years & $51.46 \pm 4.26 *$ & $63.14 \pm 8.76$ & $0.000 I^{\ddagger}$ & $50.77 \pm 10.65$ & 0.112 \\
\hline \multicolumn{6}{|l|}{ Ethnicity (\%) } \\
\hline African Americans & $380(38)$ & $387(34.4)$ & 0.1367 & $86(34.4)$ & 0.3066 \\
\hline Hispanics & $620(62)$ & $548(65.6)$ & 0.1367 & $164(65.6)$ & 0.3066 \\
\hline Male sex, n (\%) & $336(33.6)$ & $306(36.6)$ & 0.18 & $73(29.2)$ & 0.200 \\
\hline Full-dose preparation protocol & $925(92.5)$ & $778(93.1)$ & 0.587 & $230(92)$ & 0.789 \\
\hline \multicolumn{6}{|l|}{ Comorbidities $^{\Omega} \mathrm{n}(\%)$} \\
\hline Diabetes & $258(25.8)$ & 300 (35.9) & $0.0035^{\ddagger}$ & $46(18.4)$ & 0.100 \\
\hline CVA & $19(1.9)$ & $18(2.1)$ & 0.740 & $4(1.6)$ & 1.000 \\
\hline Cirrhosis & II (I.I) & $12(1.4)$ & 0.534 & $2(0.8)$ & 1.000 \\
\hline CKD & $68(6.8)$ & $60(7.2)$ & 0.782 & $15(6)$ & 0.776 \\
\hline Dementia & $6(0.6)$ & $6(0.7)$ & 0.778 & I $(0.4)$ & 1.000 \\
\hline Constipation & $212(21.2)$ & $49(19.6)$ & 0.6029 & $177(2 \mid .2)$ & 1.0000 \\
\hline \multicolumn{6}{|l|}{ Medications ${ }^{\epsilon} \mathrm{n}(\%)$} \\
\hline BB & $214(2 \mid .4)$ & $305(36.5)$ & $0.000 I^{\ddagger}$ & $41(16.4)$ & 0.08 \\
\hline$C C B$ & $224(22.4)$ & $237(28.3)$ & $0.0035^{\ddagger}$ & $44(17.6)$ & 0.10 \\
\hline Opiates & $264(26.4)$ & $240(28.7)$ & 0.2701 & $56(22)$ & 0.22 \\
\hline $\mathrm{TCA}$ & $61(6.1)$ & $48(5.7)$ & 0.7672 & $17(6.8)$ & 0.66 \\
\hline
\end{tabular}

Notes: *Plus-minus values are the mean \pm standard deviation; ${ }^{*}<0.05$ was considered statistically significant; ${ }^{\epsilon}$ comorbidities were not mutually exclusive, with any one patient possibly having multiple comorbidities; ${ }^{\Omega}$ medications were not mutually exclusive, with any one patient possibly using multiple medications.

Abbreviations: BB, beta-blockers; CCB, calcium channel blockers; CKD, chronic kidney disease; CVA, cerebrovascular accident; TCA, tricyclic antidepressants.

cerebrovascular accident, and use of prescription medications, including beta-blockers, calcium channel blockers, opiates, and tricyclic antidepressants.

\section{Definitions}

\section{Full-dose protocol}

The full-dose protocol involved administration of the entire volume of the polyethylene glycol-based formulation in the evening before the colonoscopy. After this, the patients were asked to fast until completion of colonoscopy.

\section{Split-dose protocol}

The split-dose protocol involved administration of half of the polyethylene glycol-based formulation in the evening before the colonoscopy and the remaining half on the morning of the colonoscopy. Patients were asked to fast for at least 2 hours

Table 2 Quality of bowel preparation in patients classified based on risk of colorectal cancer before colonoscopy

\begin{tabular}{|c|c|c|c|c|c|}
\hline $\begin{array}{l}\text { Quality } \\
\text { of bowel } \\
\text { oreparation }\end{array}$ & $\begin{array}{l}\text { Group I } \\
\text { total n, } \\
(\%)\end{array}$ & $\begin{array}{l}\text { Group } 2 \\
\text { total } n \text {, } \\
(\%)\end{array}$ & $\begin{array}{l}P \text {-value } \\
\text { Groups } \\
\text { I, } 2\end{array}$ & $\begin{array}{l}\text { Group } 3 \\
\text { total n, } \\
(\%)\end{array}$ & $\begin{array}{l}\text { P-value } \\
\text { Groups } \\
\mathrm{I}, 3\end{array}$ \\
\hline & & 379 & & & \\
\hline & & & & & \\
\hline & 6) & 149 & & 30 & \\
\hline adec & $676(67.6)^{€}$ & $456(54.6)^{€}$ & $0.000 I^{\ddagger}$ & $97(38.8)^{\epsilon}$ & $0.0001 \ddagger$ \\
\hline
\end{tabular}

Notes: $\$ P<0.05$ was considered statistically significant; ${ }^{\epsilon}$ total number of inadequate bowel preparations was the sum of numbers of fair and poor bowel preparations. after ingestion of the clear liquid, as per American Society for Anesthesiology guidelines.

\section{Good bowel preparation}

Good bowel preparation was defined as the absence of or only a minimal amount of solid stool with or without coexistent clear fluid that required suctioning.

\section{Fair bowel preparation}

Fair bowel preparation was defined as the presence of semisolid debris that was cleared with difficulty.

\section{Poor bowel preparation}

Poor bowel preparation was defined as the presence of solid or semisolid debris that could not be cleared effectively, thus precluding adequate visualization.

\section{Inadequate bowel preparation}

Inadequate bowel preparation was defined as a combination of fair and poor bowel preparations.

\section{Evaluation of results}

We attempted to find differences in the quality of bowel preparations between the three study groups after matching for potential confounding factors such as sex, preferred language of instructions, ethnicity, comorbidities (diabetes and cerebrovascular accident), and use of colonic motility 


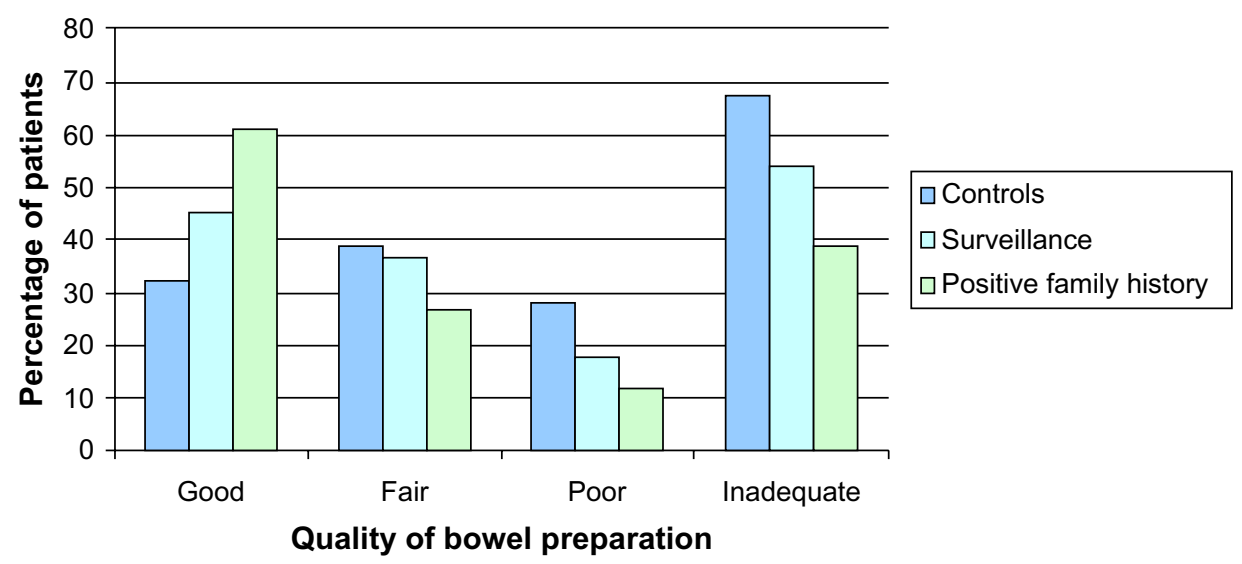

Figure I Quality of bowel preparation in patients classified based on the risk of colorectal cancer before colonoscopy.

inhibitors (beta-blockers, calcium channel blockers, opiates, and tricyclic antidepressants).

\section{Statistical analysis}

Continuous variables were expressed as the mean and standard deviation, and categorical variables were expressed as percentages. Fisher's Exact test was used to analyze the distribution of categorical data, and analysis of variance was used to compare the means. $P$-values less than 0.05 were considered to be statistically significant.

\section{Results}

In all, 7,834 patients underwent elective colonoscopy in the outpatient setting of our hospital during the study period. Of these, 834 patients showed a high risk of CRC on surveillance colonoscopy because of a personal history of adenomatous polyps observed on a previous colonoscopy (included in group 2) and 250 patients showed a high risk of CRC on screening colonoscopy because of their family history of $\mathrm{CRC}$ in first-degree relatives (included in group 3). In addition, 1,000 subjects matched for age, sex and ethnicity who were attending their first screening colonoscopy were selected as controls (included in group 1).

Baseline characteristics of the three groups were compared (Table 1). No statistically significant difference was observed between the mean age of controls (51.46 \pm 4.26 years) and that of patients with a family history of CRC $(50.77 \pm 10.65$ years; $P=0.112$ ). However, patients undergoing surveillance colonoscopy because of a personal history of adenomatous polyps (mean age $63.14 \pm 8.76$ years) were significantly older than controls $(P=0.0001)$. Distribution according to ethnicity and sex was not significantly different between the three groups. The majority of patients in the three groups underwent full-dose bowel preparation before the colonoscopy, and the differences were not statistically significant. Significantly more patients in the surveillance colonoscopy group had diabetes than in the control group $(P=0.0035)$. However, the distribution of diabetes among patients with a family history of CRC was similar to that in controls $(P=0.100)$. Use of both beta-blockers and calcium channel blockers was higher among patients in the surveillance colonoscopy group than in the control group ( $P=0.0001$ and $P=0.0035$, respectively). Use of these medications was not significantly different among patients with a family history of CRC compared with controls ( $P=0.08$ and $P=0.10$, respectively).

The quality of bowel preparation in each group is shown in Table 2. A significantly greater proportion of patients in the surveillance colonoscopy group had good-quality bowel preparation (adequate examination) than those in the control group $(P=0.0001)$. Similar results were obtained for patients with a family history of $\mathrm{CRC}(P=0.0001)$. Both fair-quality and poor-quality bowel preparations were considered inadequate because potentially premalignant lesions could not be conclusively excluded (Figure 1).

We performed a subgroup analysis to determine whether these differences were seen individually in each ethnic population. In both Hispanics (Table 3, Figure 2) and African

Table 3 Quality of bowel preparation in Hispanic patients classified based on risk of colorectal cancer before colonoscopy

\begin{tabular}{llllll}
\hline $\begin{array}{l}\text { Quality } \\
\text { of bowel } \\
\text { preparation }\end{array}$ & $\begin{array}{l}\text { Group I } \\
\text { total n, }\end{array}$ & $\begin{array}{l}\text { Group 2 } \\
\text { total n, }\end{array}$ & $\begin{array}{l}\text { P-value } \\
\text { Groups }\end{array}$ & $\begin{array}{l}\text { Group 3 } \\
\text { total n, } \\
\text { I\%) }\end{array}$ & $\begin{array}{l}\text { P-value } \\
\text { Groups } \\
\text { I, 3 }\end{array}$ \\
\hline Good & $198(31.9)$ & $252(45.9)$ & & $105(64.0)$ & \\
Fair & $242(39.0)$ & $200(36.4)$ & $0.000 I^{\ddagger}$ & $38(23.1)$ & $0.000 I^{\ddagger}$ \\
Poor & $180(29.0)$ & $96(17.5)$ & & $21(12.8)$ & \\
Inadequate & $422(68.0)^{€}$ & $296(54.0)^{€}$ & $0.000 I^{\ddagger}$ & $59(35.9)^{€}$ & $0.000 I^{\ddagger}$ \\
\hline
\end{tabular}

Notes: $¥ P<0.05$ was considered statistically significant; ${ }^{\epsilon_{\text {total }}}$ number of inadequate bowel preparations was the sum of numbers of fair and poor bowel preparations. 


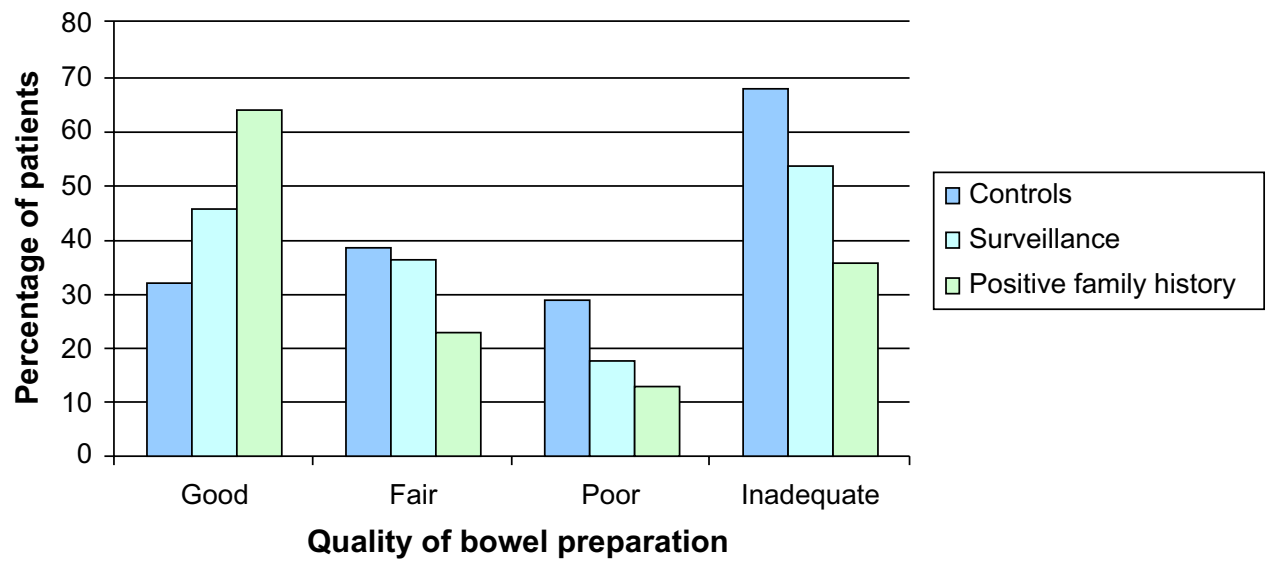

Figure 2 Quality of bowel preparation in Hispanic patients classified based on the risk of colorectal cancer before colonoscopy.

Americans (Table 4, Figure 3), the quality of bowel preparation was significantly different.

The adenoma detection rate was significantly higher in patients with a personal history of adenomatous polyps and in those with a family history of CRC than in patients undergoing routine screening colonoscopy $(P=0.0001$; Table 5$)$.

\section{Discussion}

The quality of bowel preparation is influenced by multiple factors that are dependent on patients and bowel preparation protocols. Multiple studies have shown that age is an independent predictor of the quality of bowel preparation, ${ }^{12,15}$ with advanced age having a negative influence on the quality of bowel preparation. In our study, patients undergoing surveillance colonoscopy were significantly older than controls, for individual ethnicity as well as for the combined population. However, a significantly higher proportion of patients undergoing surveillance colonoscopy had adequate bowel preparation when compared with controls. Subgroup analysis showed that this was true for both ethnicities. Older patients in the surveillance colonoscopy group could not be matched with those in the control group who underwent routine screening

Table 4 Quality of bowel preparation in African American patients classified based on the risk of colorectal cancer before colonoscopy

\begin{tabular}{|c|c|c|c|c|c|}
\hline $\begin{array}{l}\text { Quality } \\
\text { of bowel } \\
\text { oreparation }\end{array}$ & $\begin{array}{l}\text { Group I } \\
\text { total n, } \\
(\%)\end{array}$ & $\begin{array}{l}\text { Group } 2 \\
\text { total n, } \\
(\%)\end{array}$ & $\begin{array}{l}\text { P-value } \\
\text { Groups } \\
1,2\end{array}$ & $\begin{array}{l}\text { Group } 3 \\
\text { total n, } \\
(\%)\end{array}$ & $\begin{array}{l}\text { P-value } \\
\text { Groups } \\
\mathrm{I}, 3\end{array}$ \\
\hline & & & & & \\
\hline & & & & & $0.000 \mathrm{I}^{\ddagger}$ \\
\hline & & 53 & & & \\
\hline Inadec & $254(66.8)^{€}$ & $160(55.7)^{€}$ & $0.0037^{\ddagger}$ & $38(44.1)^{€}$ & $0.000 \mathrm{I}^{\ddagger}$ \\
\hline
\end{tabular}

Notes: $¥ P<0.05$ was considered statistically significant; ${ }^{\epsilon}$ total number of inadequate bowel preparations was the sum of numbers of fair and poor bowel preparations. colonoscopy because the majority of these control patients had undergone their first ever colonoscopy. The remaining baseline demographic variables (ethnicity and sex) were distributed similarly among the three groups, either individually for each ethnicity or for the combined population.

Multiple randomized controlled trials have shown that split-dose bowel preparation improves patient compliance with precolonoscopy instructions and outcomes of colonoscopy. ${ }^{19-21}$ In our study, only a small fraction of patients in each study group underwent split-dose bowel preparation. The use of this protocol was similar across all the study groups for both ethnicities. We examined the most common comorbidities, including diabetes, cirrhosis, and cerebrovascular accident, ${ }^{13}$ which adversely affected the quality of bowel preparation. Studies have shown that diabetes causes colonic dysmotility secondary to the loss of enteric neurons because of increased autonomic dysfunction and oxidative stress. ${ }^{16,22}$ We observed that the prevalence of diabetes was significantly higher in the surveillance colonoscopy group than in the control group, which could be attributed to the advanced age of patients in the former group. However, this did not influence the quality of bowel preparation in either Hispanic or African American patients. In addition, we assessed the use of common colon motility inhibitors, such as beta-blockers, calcium channel blockers, opiates, and tricyclic antidepressants, in each study group and ethnicity, and found that use of these medications was significantly greater among patients in the surveillance group, which again could be attributed to an increased disease burden among these patients due to advancing age. However, this increase in usage did not affect the quality of bowel preparation among patients in the surveillance group. This was observed for both ethnicities included in this study. We observed that the percentages of inadequate quality of bowel preparation were higher in the 


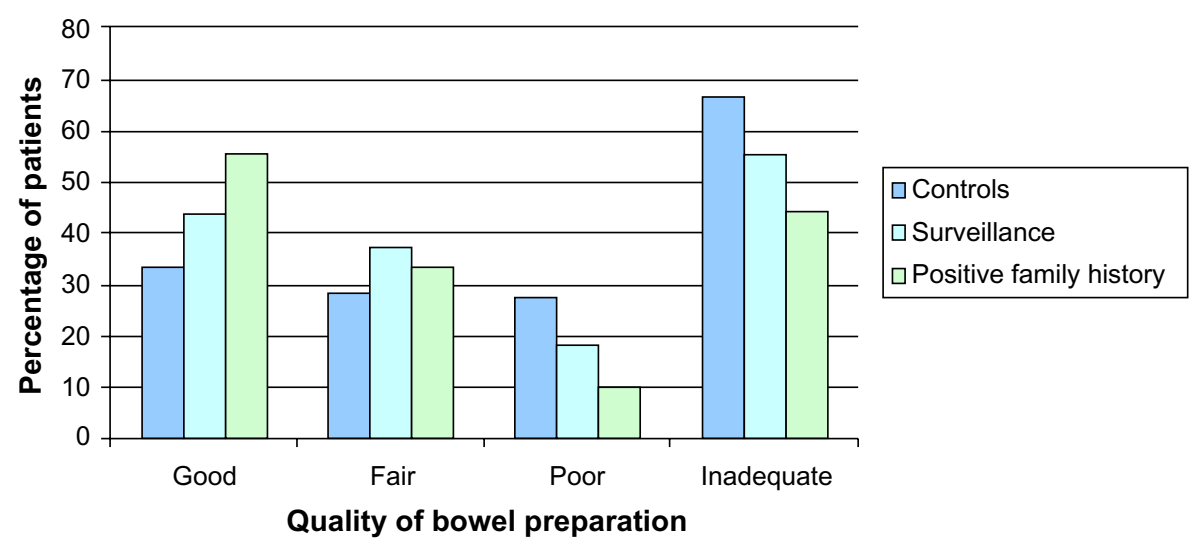

Figure 3 Quality of bowel preparation in African American patients classified based on the risk of colorectal cancer before colonoscopy.

control group for both the ethnicities compared with those observed in other studies. This could be partly attributed to differences in prevalence of known risk factors for poor bowel preparation among different ethnicities. These differences between studies could be because of wide heterogeneity in bowel preparation modalities and protocols. The rationale for screening and surveillance colonoscopy was clearly explained to all patients considered as having a high risk of CRC. Adherence to the bowel preparation regimen was strongly emphasized at the time of scheduling the colonoscopy. The same was done for patients who were considered to have an average risk of CRC. Therefore, the finding of a significantly higher number of adequate bowel preparations among patients with a high risk of CRC (irrespective of ethnicity) highlights the influence of perception of cancer risk on the behavioral patterns of patients. It could be argued that patients with a high risk of CRC could have received verbal instructions of better quality and that their perception of high risk of CRC could have improved their adherence to dietary restrictions and bowel preparation regimens.

Our study has some limitations that are inherent to its retrospective design. There is considerable heterogeneity in grading the quality of bowel preparation by different endoscopists. It has been noted in previous studies ${ }^{23}$ that the timing of colonoscopy does not influence the quality of bowel

Table 5 Adenoma detection rate in the three study groups

\begin{tabular}{llllll}
\hline Variable & $\begin{array}{l}\text { Group I } \\
\text { total n, } \\
\text { (\%) }\end{array}$ & $\begin{array}{l}\text { Group 2 } \\
\text { total n, } \\
(\%)\end{array}$ & $\begin{array}{l}\text { P-value } \\
\text { Groups } \\
\mathbf{I}, \mathbf{2}\end{array}$ & $\begin{array}{l}\text { Group 3 } \\
\text { total n, } \\
(\%)\end{array}$ & $\begin{array}{l}\text { P-value } \\
\text { Groups } \\
\text { I, 3 }\end{array}$ \\
\hline $\begin{array}{l}\text { Adenoma } \\
\text { detection }\end{array}$ & $220(22)$ & $96(38.4)$ & $0.000 \mathrm{I}^{\ddagger}$ & $280(33.6)$ & $0.000 \mathrm{I}^{\ddagger}$ \\
rate (\%) & & & & & \\
\hline
\end{tabular}

Note: $¥ P<0.05$ was considered statistically significant. preparation. However, multiple studies have shown that the duration of sip to scope time significantly affects the quality of bowel preparation. ${ }^{23-25}$ We did not have sufficient data on the timing of colonoscopies, which might have biased our results. In addition, there was an inherent selection bias among patients undergoing surveillance colonoscopies because they were aware of bowel preparation regimens and the procedure itself. It could be argued that these patients had a better understanding of the diet and bowel preparation regimens than colonoscopy-naïve patients. All the patients were provided with written instructions to reinforce the salient features of dietary restrictions and bowel preparation regimens; however, we did not have information on their education or socioeconomic status. Both these factors have been found to affect the quality of bowel preparation.

In conclusion, we found that perception of CRC risk influenced patient behavior, leading to a significantly higher number of adequate bowel preparations among patients having a higher risk of CRC than in those undergoing routine screening colonoscopy. These differences were statistically significant for both Hispanic and African American patients.

\section{Author contributions}

All authors made substantial contributions to conception and design, acquisition of data, drafting the article and revising it critically for important intellectual content, gave final approval of the published version and agree to be accountable for all aspects of the work.

\section{Disclosure}

None of the authors have a direct financial relationship with any commercial identities mentioned in the paper. The authors report no conflicts of interest in this work. 


\section{References}

1. Siegel R, Naishadham D, Jemal A. Cancer statistics, 2013. CA Cancer J Clin. 2013;63(1):11-30.

2. Centers for Disease Control and Prevention. Vital signs: colorectal cancer screening, incidence, and mortality - United States, 2002-2010. MMWR Morb Mortal Wkly Rep. 2011;60(26):884-889.

3. Edwards BK, Ward E, Kohler BA, et al. Annual report to the nation on the status of cancer, 1975-2006, featuring colorectal cancer trends and impact of interventions (risk factors, screening, and treatment) to reduce future rates. Cancer. 2010;116(3):544-573.

4. Brenner H, Hoffmeister M, Arndt V, Stegmaier C, Altenhofen L, Haug U. Protection from right- and left-sided colorectal neoplasms after colonoscopy: population-based study. J Natl Cancer Inst. 2010; 102(2):89-95.

5. Baxter NN, Goldwasser MA, Paszat LF, Saskin R, Urbach DR, Rabeneck L. Association of colonoscopy and death from colorectal cancer. Ann Intern Med. 2009;150(1):1-8.

6. Nishihara R, Wu K, Lochhead P, et al. Long-term colorectal-cancer incidence and mortality after lower endoscopy. N Engl J Med. 2013; 369(12):1095-1105.

7. Ransohoff DF. How much does colonoscopy reduce colon cancer mortality? Ann Intern Med. 2009;150(1):50-52.

8. Heitman SJ, Ronksley PE, Hilsden RJ, Manns BJ, Rostom A, Hemmelgarn BR. Prevalence of adenomas and colorectal cancer in average risk individuals: a systematic review and meta-analysis. Clin Gastroenterol Hepatol. 2009;7(12):1272-1278.

9. Ramsey SD, Yoon P, Moonesinghe R, Khoury MJ. Population-based study of the prevalence of family history of cancer: implications for cancer screening and prevention. Genet Med. 2006;8(9):571-575.

10. Winawer SJ, Fletcher RH, Miller L, et al. Colorectal cancer screening: clinical guidelines and rationale. Gastroenterology. 1997;112(2): 594-642.

11. Ioannou GN, Chapko MK, Dominitz JA. Predictors of colorectal cancer screening participation in the United States. Am J Gastroenterol. 2003;98(9):2082-2091.

12. Nguyen DL, Wieland M. Risk factors predictive of poor quality preparation during average risk colonoscopy screening: the importance of health literacy. J Gastrointestin Liver Dis. 2010;19(4):369-372.

13. Ness RM, Manam R, Hoen H, Chalasani N. Predictors of inadequate bowel preparation for colonoscopy. Am J Gastroenterol. 2001;96(6): 1797-1802.
14. Chan WK, Saravanan A, Manikam J, Goh KL, Mahadeva S Appointment waiting times and education level influence the quality of bowel preparation in adult patients undergoing colonoscopy. BMC Gastroenterol. 2011;11:86.

15. Chung YW, Han DS, Park KH, et al. Patient factors predictive of inadequate bowel preparation using polyethylene glycol: a prospective study in Korea. J Clin Gastroenterol. 2009;43(5):448-452.

16. Battle WM, Snape WJ Jr, Alavi A, Cohen S, Braunstein S. Colonic dysfunction in diabetes mellitus. Gastroenterology. 1980;79(6): 1217-1221.

17. Serper M, Gawron AJ, Smith SG, et al. Patient factors that affect quality of colonoscopy preparation. Clin Gastroenterol Hepatol. 2014;12(3): $451-457$.

18. Smith SG, von Wagner C, McGregor LM, et al. The influence of health literacy on comprehension of a colonoscopy preparation information leaflet. Dis Colon Rectum. 2012;55(10):1074-1080.

19. Kilgore TW, Abdinoor AA, Szary NM, et al. Bowel preparation with split-dose polyethylene glycol before colonoscopy: a meta-analysis of randomized controlled trials. Gastrointest Endosc. 2011;73(6): 1240-1245.

20. Enestvedt BK, Tofani C, Laine LA, Tierney A, Fennerty MB. 4-Liter split-dose polyethylene glycol is superior to other bowel preparations, based on systematic review and meta-analysis. Clin Gastroenterol Hepatol. 2012;10(11):1225-1231.

21. Cohen B, Tang RS, Groessl E, Herrin A, Ho SB. Effectiveness of a simplified "patient friendly" split dose polyethylene glycol colonoscopy prep in Veterans Health Administration patients. J Interv Gastroenterol. 2012;2(4):177-182.

22. Chandrasekharan B, Anitha M, Blatt R, et al. Colonic motor dysfunction in human diabetes is associated with enteric neuronal loss and increased oxidative stress. Neurogastroenterol Motil. 2011;23(2):131-138.

23. Eun CS, Han DS, Hyun YS, et al. The timing of bowel preparation is more important than the timing of colonoscopy in determining the quality of bowel cleansing. Dig Dis Sci. 2011;56(2):539-544.

24. Gurudu SR, Ratuapli S, Heigh R, DiBaise J, Leighton J, Crowell M. Quality of bowel cleansing for afternoon colonoscopy is influenced by time of administration. Am J Gastroenterol. 2010;105(11):2318-2322.

25. Cohen LB, Kastenberg DM, Mount DB, Safdi AV. Current issues in optimal bowel preparation: excerpts from a roundtable discussion among colon-cleansing experts. Gastroenterol Hepatology. 2009; 5(11 Suppl 19):1-11.
Clinical and Experimental Gastroenterology

\section{Publish your work in this journal}

Clinical and Experimental Gastroenterology is an international, peerreviewed, open access journal, publishing all aspects of gastroenterology in the clinic and laboratory, including: Pathology, pathophysiology of gastrointestinal disease; Investigation and treatment of gastointestinal disease; Pharmacology of drugs used in the alimentary tract;

\section{Dovepress}

Immunology/genetics/genomics related to gastrointestinal disease. This journal is indexed on CAS. The manuscript management system is completely online and includes a very quick and fair peer-review system. Visit http://www.dovepress.com/testimonials.php to read real quotes from published authors. 